\title{
Accurate Temporal Discretization of Time Domain Boundary Integral Equations
}

\author{
Yves Beghein \\ Kristof Cools \\ Daniël De Zutter \\ Ghent University, Belgium \\ University of Nottingham, UK \\ Ghent University, Belgium \\ Email: yves.beghein@ugent.be Email: kristof.cools@nottingham.ac.uk Email:daniel.dezutter@intec.ugent.be
}

\begin{abstract}
In this contribution, a novel temporal discretization scheme for time domain boundary integral equations is introduced. It distinguishes itself by (i) a new approach to the construction of higher order temporal basis functions, and (ii) the use of temporal PetrovGalerkin testing as opposed to the widespread collocation in time method. The retarded potential boundary integral equation and its classic collocation in time discretization will be revisited. Next, the new temporal basis and testing functions will be introduced. The space-time PetrovGalerkin discretization using these functions will be elucidated. Finally, numerical results are presented testifying to the improved accuracy of the novel scheme.
\end{abstract}

\section{INTRODUCTION}

Broadband scattering by perfectly conducting bodies can be modeled by time domain boundary integral equations, which can be solved numerically using the marching-on-in-time (MoT) algorithm. The most frequently used approach is the collocation in time method, in which the signals are approximated by the so-called shifted Lagrange interpolants [1] and the equation is tested by evaluating it at subsequent time steps [2].

In contrast to this, this contribution proposes a new set of temporal basis functions, in combination with a temporal Petrov-Galerkin testing procedure. This approach greatly improves the accuracy of the results. As discretization errors are a known source of instabilities [3], this novel discretization scheme will also benefit the stability of the MoT algorithm.

\section{Discretization Scheme}

\section{A. The Marching-on-in-Time Algorithm}

Consider a perfectly conducting scatterer $\Omega$, illuminated by an incident electric field $e^{i}$ and magnetic field $\boldsymbol{h}^{i}$. On the surface $\Gamma$, the current $\boldsymbol{j}$ must be determined such that the corresponding field satisfies the boundary condition for the electric and magnetic field and linear combinations thereof, yielding the electric field, magnetic field, and combined field integral equations (EFIE, MFIE, and CFIE) respectively:

$$
\mathcal{O}(\boldsymbol{j})=\boldsymbol{x}\left(\boldsymbol{e}^{i}, \boldsymbol{h}^{i}\right)
$$

where $\mathcal{O}$ is a linear combination of the EFIE and MFIE operators, and $\boldsymbol{x}$ is a linear combination of $\boldsymbol{e}^{i}$ and $\boldsymbol{h}^{i}$. As a first step, $j$ is approximated by an expansion in a set of spatial basis functions (e.g. the RWG functions $\boldsymbol{f}_{i}(\boldsymbol{r})$ ) and a set of temporal basis functions $T_{i}(t)=T(t-i \Delta t)$ (usually, the shifted Lagrange interpolants [1]).

In the collocation in time method, (1) is evaluated at $t=i \Delta t$ and spatially tested with $\boldsymbol{g}_{j}(\boldsymbol{r})$. If $T(t)=0$ $\forall t<-\Delta t$, a causal discrete system is obtained, which can be cast into the following matrix form:

$$
\mathbf{Z}^{(0)} \mathbf{j}^{(i)}=\mathrm{x}^{(i)}-\sum_{l=0}^{i-1} \mathbf{Z}^{(i-l)} \mathbf{j}^{(l)},
$$

where $\mathrm{j}^{(l)}$ contains the RWG expansion coefficients for the current at $t=l \Delta t$. Equation (2) can be solved successively for $i=1,2$, etc. This procedure is known as the marching-on-in-time (MoT) algorithm [2].

\section{B. Higher Order Temporal Petrov-Galerkin Testing}

The temporal finite element space is now spanned by $p$ sets of basis functions $T_{l}^{(m)}(t)=T^{(m)}(t-l \Delta t), m=$ $1, \ldots, p . T^{(m)}(t)$ is the temporal basis function that is piecewise polynomial of degree $p$, globally continuous, and interpolating at $t=(m / p-1) \Delta t$. Thus, there are $p$ degrees of freedom per time step.

Next, a temporal Petrov-Galerkin procedure is applied: (1) is multiplied by a spatial as well as a temporal testing function, and integrated over both space and time. $p$ sets of testing functions $U_{i}^{(m)}(t)=U^{(m)}(t-i \Delta t)$ are needed to arrive at a square system matrix.

In general, this procedure does not allow a timemarching solution. However, if $T^{(m)}(t)=0 \forall t<-\Delta t$, and $U(t)=0 \forall t>0$, discrete causality is guaranteed. The time-marching equation then becomes: 



Fig. 1. Temporal basis functions $T^{(1)}(t)$ and $T^{(2)}(t)$ (top) and temporal testing functions $U^{(1)}(t)$ and $U^{(2)}(t)$ (bottom).

$$
\mathbf{Z}^{(0)}\left(\begin{array}{c}
\mathrm{j}_{1}^{(i)} \\
\mathrm{j}_{2}^{(i)} \\
\ldots
\end{array}\right)=\left(\begin{array}{c}
\mathrm{x}_{1}^{(i)} \\
\mathrm{x}_{2}^{(i)} \\
\ldots
\end{array}\right)-\sum_{l=0}^{i-1} \mathbf{Z}^{(i-l)}\left(\begin{array}{c}
\mathrm{j}_{1}^{(l)} \\
\mathrm{j}_{2}^{(l)} \\
\ldots
\end{array}\right)
$$

with

$$
\begin{aligned}
\mathbf{Z}^{(l)} & =\left(\begin{array}{ccc}
\mathbf{Z}_{11}^{(l)} & \mathbf{Z}_{12}^{(l)} & \ldots \\
\mathbf{Z}_{21}^{(l)} & \mathbf{Z}_{22}^{(l)} & \ldots \\
\ldots & \ldots & \ldots
\end{array}\right), \\
{\left[\mathbf{Z}_{m n}^{(i)}\right]_{j k} } & =\left(\boldsymbol{g}_{j} U_{i}^{(m)}, \mathcal{O}\left(\boldsymbol{f}_{k} T^{(n)}\right)\right), \\
{\left[\mathbf{x}_{m}^{(i)}\right]_{j} } & =\left(\boldsymbol{g}_{j} U_{i}^{(m)}, \boldsymbol{x}\left(\boldsymbol{e}^{i}, \boldsymbol{h}^{i}\right)\right),
\end{aligned}
$$

and $j_{m}^{(i)}$ contains the RWG expansion coefficients of the currents at $t=(i+m / p-1) \Delta t$. A suitable choice of basis and testing functions for $p=2$ is presented in Fig. 1, and can be generalized for general degree $p$.

\section{NumERICAL RESUltS}

A perfectly electrically conducting sphere of radius $30 \mathrm{~cm}$ is illuminated by a Gaussian pulse with duration $1.25 \mathrm{~ns}$. This scattering problem is modeled by the time domain CFIE. Spatial discretization is performed using $N_{S}=3252$ RWG basis functions. First, the collocation in time scheme is applied with second degree piecewise polynomial Lagrange interpolants as basis functions. All interaction integrals are computed as in [4]. Next, the simulation is repeated using the new scheme. After applying a Fourier transform, the results are compared to the projection of the Mie series onto the RWG basis. The $H_{d i v}^{-1 / 2}$ norm of the error is shown in Fig. 2, for simulations with an equal number of temporal degrees of freedom per unit of time, and an equal polynomial degree $p=2$. The Petrov-Galerkin scheme improves the accuracy by an order of magnitude over a broad frequency band. At small time steps, the accuracy converges
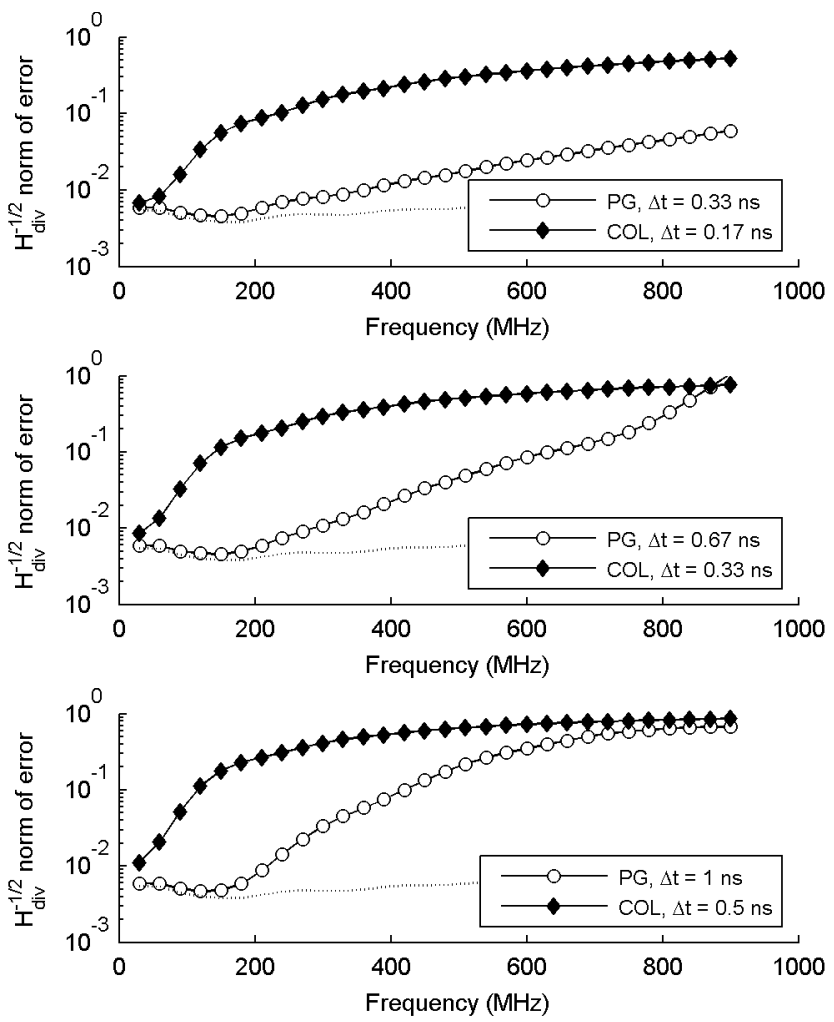

Fig. 2. $H_{d i v}^{-1 / 2}$ norm of the error with collocation in time (COL) and higher order Petrov-Galerkin (PG) for equal numbers of degrees of freedom. Dotted line: frequency domain CFIE results.

towards the accuracy of the frequency domain CFIE simulations, which is limited by the spatial discretization.

\section{CONCLUSION}

In this contribution, a new higher-order temporal Petrov-Galerkin procedure for time domain boundary integral equations was proposed. Numerical experiments have shown that this discretization scheme significantly increases the accuracy of the solution.

\section{REFERENCES}

[1] A. Geranmayeh, W. Ackermann, and T. Weiland, "Survey of temporal basis functions for integral equation methods," in Computational Electromagnetics in Time-Domain, 2007. CEMTD 2007. Workshop on, oct. 2007, pp. $1-4$.

[2] W. Chew, E. Michielssen, J. M. Song, and J. M. Jin, Eds., Fast and Efficient Algorithms in Computational Electromagnetics. Norwood, MA, USA: Artech House, Inc., 2001.

[3] F. Andriulli, K. Cools, F. Olyslager, and E. Michielssen, "Time domain Calderón identities and their application to the integral equation analysis of scattering by PEC objects part II: Stability," IEEE Trans. Antennas Propagation, vol. 57, no. 8, pp. 2365 2375, aug. 2009.

[4] B. Shanker, M. Lu, J. Yuan, and E. Michielssen, "Time domain integral equation analysis of scattering from composite bodies via exact evaluation of radiation fields," IEEE Trans. Antennas Propagation, vol. 57, no. 5, pp. 1506 -1520, may 2009. 